\title{
Reactive ion beam etching - based finishing of optical aluminium surfaces
}

\author{
Melanie Ulitschka ${ }^{1, *}$, Jens Bauer $^{1}$, Frank Frost $^{1}$, and Thomas Arnold ${ }^{1,2}$ \\ ${ }^{1}$ Leibniz Institute of Surface Engineering (IOM), D-04318 Leipzig, Germany \\ ${ }^{2}$ Technische Universität Dresden, Institute of Manufacturing Science and Engineering, D-01069 Dresden, Germany
}

\begin{abstract}
Reactively driven low-energy ion beam machining is a widely used finishing technique for optical aluminium surfaces. Direct RIBE machining with oxygen or nitrogen process gas permits figure error correction of diversely shaped aluminium optics while preserving the initial roughness up to $1 \mu \mathrm{m}$ etching depth. Those optical surfaces are appropriate for applications in the infrared spectral range. For increasing demands on optical surfaces for short - wavelength applications in the visible and ultraviolet spectral range ion beam polishing techniques with the aid of a sacrificial layer are a promising process route. Turning marks situated in the high-to - mid spatial frequency range are remarkably reduced while preserving the initial microroughness resulting in an enhancement of the full range roughness.
\end{abstract}

\section{Introduction}

For increasing demands on high-performance optical systems, scattering effects resulting from surface errors are necessary to reduce.

Technical aluminium alloy materials as Al6061 or A1905 are common mirror construction materials. For fabrication of optical aluminium surfaces conforming to the requirements in the infrared (IR) spectral range, usually single-point diamond turning (SPDT) is used [1, 2]. Periodic groove pattern microstructures (turning marks) are left on the surface having similar effects of a diffraction grating (Fig. 1).

However, the demands on optical surface quality for applications in the short-wavelength visible (VIS) and ultraviolet (UV) spectral range increase immensely. In particular, improved surface qualities with roughness values of $1 \mathrm{~nm}$ root mean square (rms) down to $0.3 \mathrm{~nm}$ are required [3].

The surface errors to be corrected are specified by the spatial frequency range they emerge $[4,5]$. Depending on the lateral dimension, the surface errors are divided into low-spatial frequency (LSF), mid-spatial frequency (MSF) and high-spatial frequency (HSF) errors.

Deviations of the surface form or shape (i.e. surface figure errors) are LSF errors. On the contrary, HSF errors are microroughness or surface finish errors causing diffusive shine. MSF errors are situated in between. Turning marks are typical feature types situated in the mid - to - high spatial frequency range of waviness and roughness (Fig. 1).

The surface errors to be corrected are on the nanometer and sub-nanometer scale. Thus ion beam technologies driven by reactive process control are a promising process route to improve the optical performance since these slow surface machining techniques offer a high degree of precision.

a)
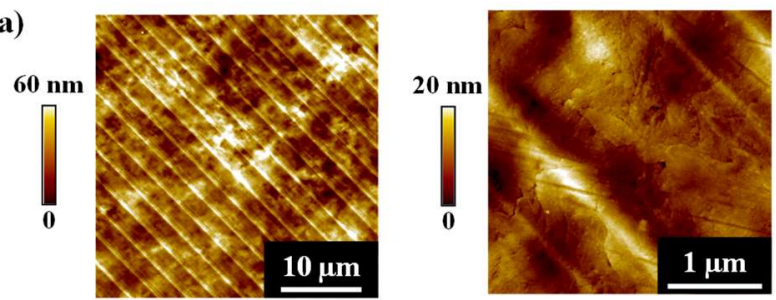

b)

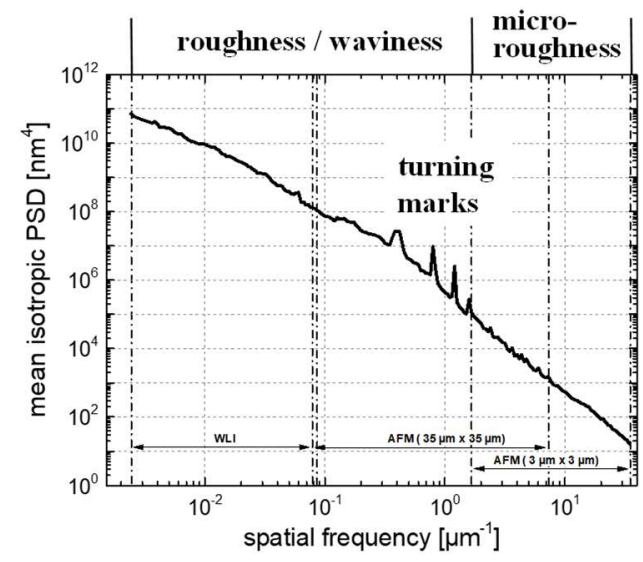

Fig. 1. Optical aluminium surface of A1905 after SPDT measured with a) Atomic force microscopy (AFM) with scan sizes of $35 \mu \mathrm{m} \times 35 \mu \mathrm{m}$ (top) and $3 \mu \mathrm{m} \times 3 \mu \mathrm{m}$ (bottom). b) Calculated power spectral density (PSD) function based on the AFM measurements depicted in a) and WLI measurements (1230 $\mu \mathrm{m}$ x $925 \mu \mathrm{m})$.

\footnotetext{
* Corresponding author: melanie.ulitschka@,iom-leipzig.de
} 


\section{Deterministic ion beam machining}

Ion beam etching technologies driven by reactive process control $\left(\mathrm{N}_{2}, \mathrm{O}_{2}\right)$ allows figure error correction of optical aluminium surfaces up to $1 \mu \mathrm{m}$ machining depth while preserving the initial surface roughness (Fig. 2b-d).

The interaction of a low- energy ion beam with the aluminium surface suggest the formation of a quasistationary surface passivation layer (Fig. 2a). The erosion process is based on pure physical sputtering. Hence, the etch rate is strongly dependent on the ion energy and the ion incidence angle $[6,7]$. a)

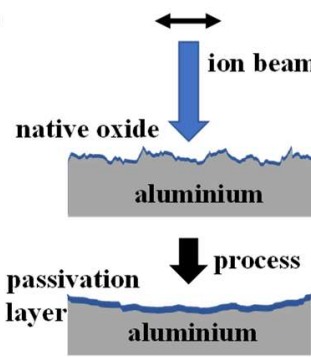

c)

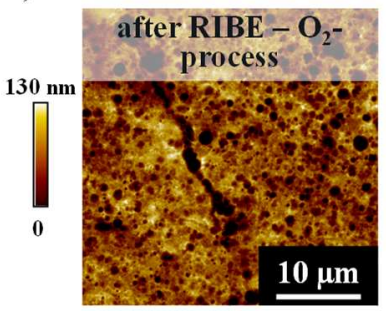

b)

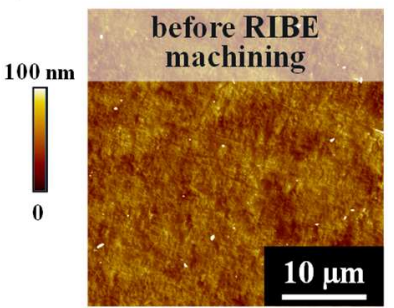

d)

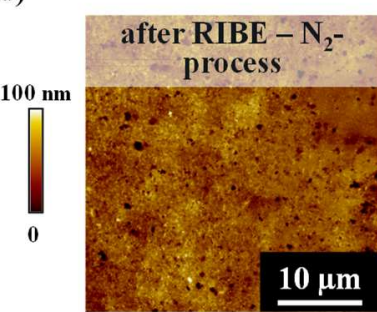

Fig. 2. a) Model scheme of RIBE techniques for figure error correction via RIBE machining with oxygen or nitrogen process gas. AFM topography images $(35 \mu \mathrm{m} \times 35 \mu \mathrm{m})$ of Al905 samples b) before RIBE machining and after ion beam machining with c) oxygen and d) nitrogen process gas.

\section{Ion beam planarization}

For ion beam planarization technique, a photoresist layer is deposited by conventional photolithography exhibiting an ultra-smooth surface. The technological thick photoresist layer fully embeds the surface roughness features of the initial aluminium surface. Removing the resist by reactive ion beam processing, the ultra-smooth surface topography of the resist is transferred into the aluminium substrate (Fig. 3a).

The selectivity, defined as the ratio of the etching rates of aluminium substrate and photoresist layer, significantly affects the planarization process. For optimized surface transfer a selectivity of 1:1 needs to be reached which can be achieved by accurately adjustment of the ion incidence angle and process gas composition.

Photoresist thermal pre-treatment was optimized to allow roughness preservation during RIBE machining and steady-state material removal rate.

During ion beam planarization the turning marks present on the initial aluminium surface are remarkably reduced in height while preserving the initial microroughness. Consequently, the full range roughness is strongly reduced (Fig. 3b-d). a)
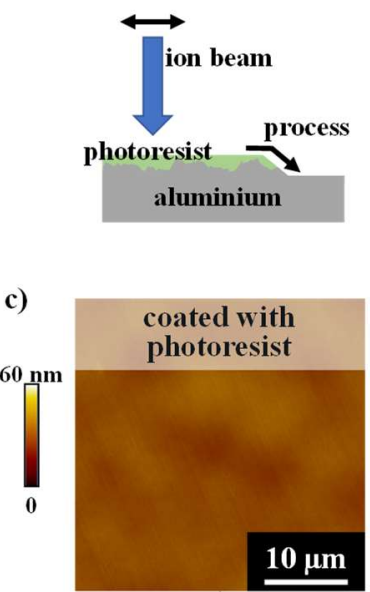

b)

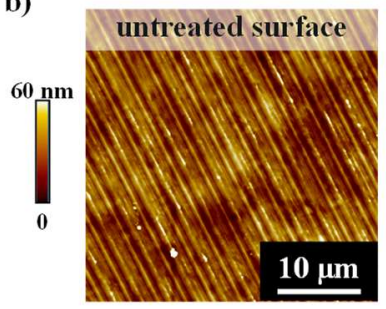

d)

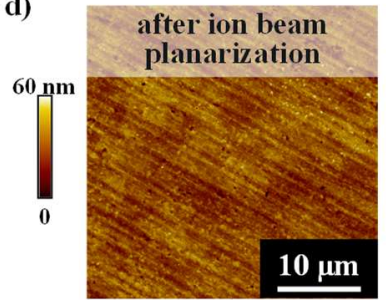

Fig. 3. a) Model scheme of RIBE techniques for planarization with the aid of a photoresist layer. AFM topography images (35 $\mu \mathrm{m} \times 35 \mu \mathrm{m})$ of $\mathrm{b})$ the untreated A1905 surface, b) coated with a photoresist layer and c) after ion beam planarization.

The authors are very grateful to Mr. T. Liebeskind for sample preparation and chemical sample treatments. Financial support by the German Federal Ministry of Education and Research (BMBF) within the framework of the InnoProfile Transfer initiative 03IPT706X 'Ultra-precision manufacturing using atomic particle beams' is gratefully acknowledged.

\section{References}

1. R. Steinkopf, A. Gebhardt, S. Scheiding, M. Rohde, O. Stenzel, S. Gliech, V. Giggel, H. Löscher, G. Ullrich, P. Rucks, A. Duparre, S. Risse, R. Eberhardt, A. Tünnermann, Proc. SPIE 7102, Optical Fabrication, Testing, and Metrology III, 71020C (2008)

2. G. P. H. Gubbels, B. W. H. Venrooy, R. Henselmans, Proc. SPIE 7426, Optical Manufacturing and Testing VIII, 742607 (2009)

3. M. Weiser, Nucl. Instruments Methods Phys. Res. Sect. B Beam Interact. With Mater. Atoms, 267, 1390-1393 (2009)

4. J. E. Harvey, A. Kotha, Proc. SPIE - The International Society for Optical Engineering 2576, 155-174 (1995)

5. J. M. Bennett, Meas. Sci. Technol. 3, 1119-1127 (1992)

6. J. Bauer, M. Ulitschka, F. Frost, T. Arnold, EOS Opt. Technol. / 5th Conf. Manuf. Opt. Syst., (European Optical Society, Munich / Germany 2017)

7. J. Bauer, F. Frost, T. Arnold, J. Phys. D. Appl. Phys. 50, 85101 (2017) 